\title{
In situ STEM Mechanical Experiments at Atomic-Resolution Using a MEMS Device
}

Eita Tochigi ${ }^{*}{ }^{*}$, Takaaki Sato ${ }^{2}$, Naoya Shibata ${ }^{1,3}$, Hiroyuki Fujita $^{4}$ and Yuichi Ikuhara ${ }^{1,3,5}$

1. Institute of Engineering Innovation, The University of Tokyo, Tokyo, Japan.

2. Department of Mechanical Engineering and Applied Mechanics, University of Pennsylvania, Philadelphia, USA.

3. Nanostructures Research Laboratory, Japan Fine Ceramics Center, Nagoya, Japan.

4. Advanced Research Laboratories, Tokyo City University, Tokyo, Japan.

5. Center for Elements Strategy Initiative for Structure Materials, Kyoto University, Kyoto, Japan.

* Corresponding author: tochigi@sigma.t.u-tokyo.ac.jp

In situ transmission electron microscopy (TEM) mechanical test is a powerful technique to directly observe microstructural evolution of crystalline materials upon loading. This technique can visualize the dynamic behavior of lattice defects, such as dislocation motion, twinning, or stress-induced phase transformation, at nanometer scale. These phenomena are essentially associated with atomic structure changes, and thus it is desirable to extend the technique to atomic scale. However, this is still challenge because of high demands laid on in situ TEM sample holders: (1) precise machinery for nanomechanical test, (2) double-tilt capability to align crystal orientation, (3) compatibility with narrow-gap pole pieces for atomic-resolution TEM. To satisfy these demands, miniaturized mechanical actuators are indispensable. Microelectromechanical systems (MEMS) technology is useful to fabricate electrical circuits, sensors, or mechanical actuators in micrometer order. MEMS devices have been applied to in situ TEM mechanical experiments [1], but there are few reports on in situ experiments under atomic- resolution observation. In this study, we developed a customized MEMS device for nanomechanical test compatible with a commercially available biasing TEM holder. Using this system, we demonstrate in situ mechanical test at atomic-resolution performed in an aberration-corrected scanning TEM (STEM).

Figure 1 shows a MEMS device for nanomechanical test developed in this study, which is compatible with a double-tilt biasing holder (Aduro, Protochips). This device was made from a silicon on insulator (SOI) wafer by standard lithography processes and focused ion beam (FIB). The two beams are driven as electrostatic actuator by the electrical potential difference between the beams and the electrodes. The vertical beam moves to the right-hand direction and the horizontal beam moves along the vertical direction. Their stiffness is $30 \mathrm{~N} / \mathrm{m}$ and $3.1 \mathrm{~N} / \mathrm{m}$, respectively. The operating principles of this device is given elsewhere [2].

A testing sample with the size of about $20 \times 10 \times 2 \mu \mathrm{m}^{3}$ was cut, lifted-out and mounted to bridge the two beams of the MEMS device by FIB (Helios G4 UX, Thermo Fisher Scientific). The sample was further cut to a shape suitable for a mechanical test and thinned to obtain electron transparency. In situ TEM mechanical test was performed in an aberration-corrected STEM (ARM-200F, JEOL) operated at $200 \mathrm{kV}$ with an electron beam convergence semi-angle of $24 \mathrm{mrad}$.

Figure 2a shows a high angle annular dark field (HAADF) STEM image of a $\mathrm{SrTiO}_{3}$ single crystal mounted on the MEMS device without loading. The sample is perfectly oriented along the [100] zone axis. The blank part at the right center is a notch to lead stress concentration within the field of view. The strain map along the vertical direction $\left(\boldsymbol{e}_{y y}\right)$ obtained by the geometrical phase analysis $[3,4]$ is 
shown at the top left. The red-green fluctuations $(\sim \pm 1 \%)$ are likely to be due to the instability in the present experimental system. Fig. 2b shows a HAADF STEM image of the sample with an applied load of $15.4 \mu \mathrm{N}$. The atomic columns are still clearly seen. From the strain map, the tensile strain region is found to generate from the notch, indicating that mechanical test with atomic-resolution is successfully done in situ. In the presentation, we will show sequential images taken during the in situ experiment and discuss strain distribution changes and microstructural evolution at the notch with different loading conditions [4].

\section{References:}

[1] Y Zhu and HD Espinosa, Proc. Natl. Acad. Sci. U. S. A. 102 (2005), p. 14503.

[2] T Sato et al., Microelectro. Eng. 164 (2016), p. 43.

[3] MJ Hytch et al., Ultramicrosc. 74 (1998), p. 131.

[4] Strain maps were obtained using the FRWRtools plugin for Gatan DigitalMicrograph written by CT Koch (https://www.physics.hu-berlin.de/en/sem/software/software frwrtools). The authors acknowledge funding of Grant-in-Aid for Specially Promoted Research (Grant No. JP17H06094) and for Scientific Research (A) (Grant No. JP17H01049) from the Japan Society for the Promotion of Science (JSPS), the Elements Strategy Initiative for Structural Materials (ESISM) from the Ministry of Education, Culture, Sports, Science, and Technology in Japan (MEXT), and JSPS KAKENHI (Grant No. JP 18K13981)

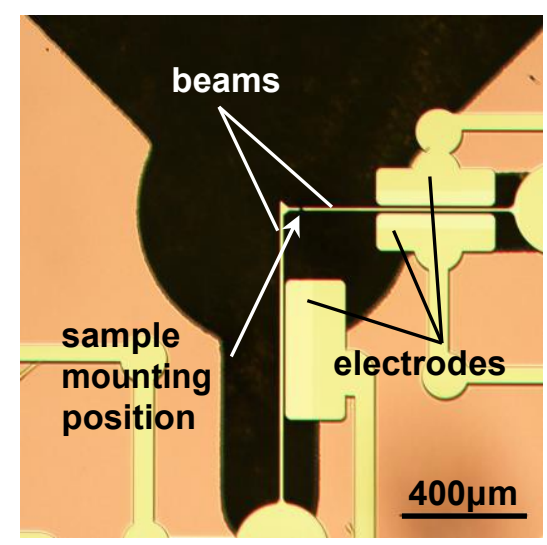

Figure 1. The guts of a MEMS device for in situ TEM mechanical test.
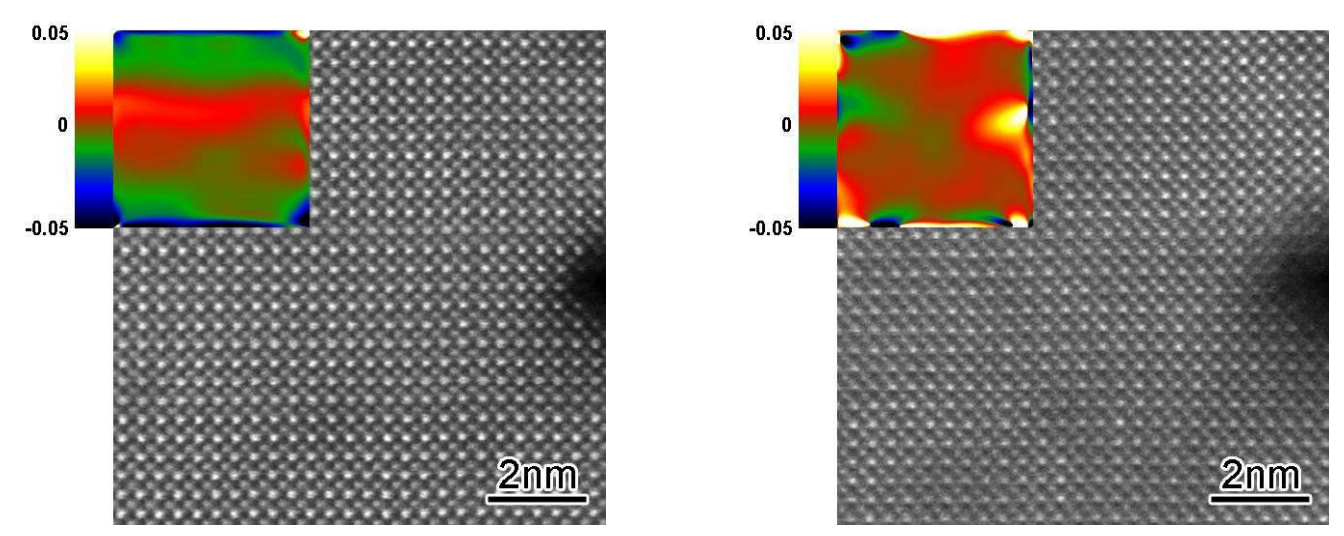

Figure 2. HAADF-STEM images and strain maps (e $\left.\boldsymbol{e}_{y y}\right)$ of (a) load-free and (b) the load of $15.4 \mu \mathrm{N}$. 